\title{
Inspiratory muscle testing in stable COPD patients
}

\author{
T. Similowski, J-P.H. Derenne
}

Inspiratory muscle testing in stable COPD patients. T. Similowski, J-Ph. Derenne. (C) ERS Journals Ltd 1994.

ABSTRACT: Exploration of inspiratory muscles in stable chronic pulmonary disease patients can be important in the investigation of a respiratory handicap unsatisfactorily explained by alterations of the passive respiratory system, or in the follow-up of patients undergoing treatments that can interfere with muscle function. Compensatory mechanisms tend to counterbalance the deleterious effects of hyperinflation in these patients, and precise clinical data are needed in order to avoid mistakes due to underverified hypotheses.

Investigation of inspiratory muscle function requires the study of output data under various states of activity of the system. As outputs, volume displacement lacks specificity, pressure measurements can be more specific but are at times invasive and should be associated with lung volume measurements, electromyography is methodologically complex, nonquantitative and of poor reproducibility. Voluntary manoeuvres depend upon subject co-operation, and do not allow partitioning of output between the action of different muscle groups. Transcutaneous electrical phrenic nerve stimulation is devoid of these inconveniences, but it explores only one muscle (the diaphragm) under conditions that are not "natural" (relaxed rib cage).

Recently, perspectives for easier clinical assessment of inspiratory muscle function in chronic obstructive pulmonary disease patients have been opened by cervical magnetic stimulation, better understanding of the meaning of mouth pressure in relationship to phrenic stimulation, and development of noninvasive tests, such as nostril pressure during sniff or phonomyography. If validated, such tests should provide a reasonably limited panel of clinical tools to better appreciate muscle function in this setting.

Eur Respir J., 1994, 7, 1871-1876.
Laboratoire de Physio-Pathologie Respiratoire, Service de Pneumologie et de Réanimation, Groupe Hospitalier PitiéSalpêtrière, Paris, France.

Correspondence: T. Similowski Groupe Hospitalier Pitié-Salpêtrière Service de Pneumologie et de Reanimation F-75651 Paris Cedex 13

France

Keywords:

Chronic obstructive pulmonary disease diaphragm

pulmonary function tests

Received: July 121994

Accepted after publication July 211994
Evaluating inspiratory muscle function in stable chronic obstructive pulmonary disease (COPD) patients is often considered a difficult task. This is clearly illustrated by a marked discrepancy between the large amount of data available in animals or healthy human volunteers under various conditions on the one hand, and the present limited knowledge in patients on the other. Several reasons can explain this phenomenon. Firstly, respiratory muscles are only one component in a complex sequence that eventually generates tidal volume to allow gas exchange (fig. 1). Therefore, isolating their contribution to the results of easy to perform, but global, tests can be difficult. Secondly, as methods tend to provide closer insights on intrinsic respiratory muscle properties, they also require more sophisticated equipment and become more delicate to operate.

As in many medical situations, choosing a method to investigate respiratory muscle characteristics and interpreting its results requires the question asked to be clearly defined, and the investigator to be aware of the limits of the test and confounding factors. This is particularly important when other systems (i.e. passive respiratory system mechanics or control of breathing) are largely abnormal, as is the case in COPD.

The present paper is not intended to be a comprehensive review of current knowledge about respiratory muscle function in COPD patients, nor does it pretend to give a complete description and critique of available tests. Rather, it aims at illustrating how the exploration of respiratory muscles navigates between "simple" (but probable "false") and "true" (but generally "complex and practically difficult to use"). It attempts to provide a brief overview of current methods and perspectives oriented toward routine clinical investigation.

\section{Diaphragm function in stable COPD [1]}

Why should respiratory muscles be explored in COPD patients? From the clinical point of view, respiratory muscle investigations are probably of little diagnostic help in the setting of a "normal" patient. They can be of more interest in situations where muscle dysfunction is highly likely to contribute to the respiratory 


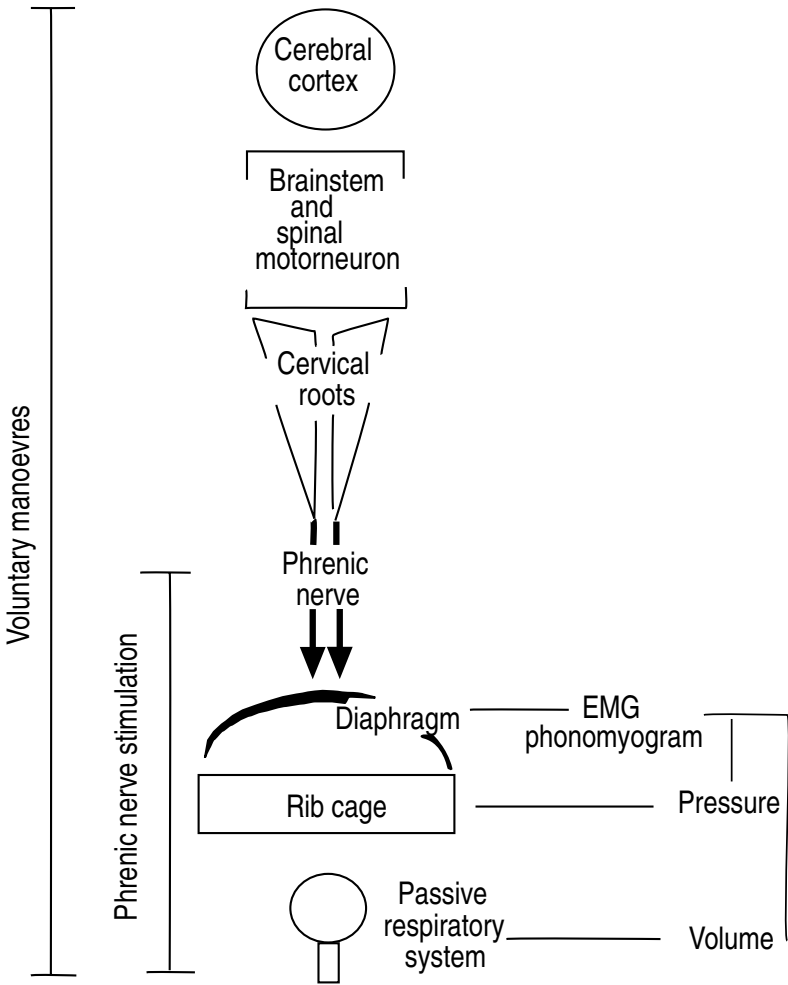

Fig. 1. - Schematic representation of the respiratory determinants of inspiratory muscle function. On the right are indicated outputs with their aggregate contributors. On the left are indicated inputs with the part of the sequence they explore. EMG: electromyogram.

handicap (e.g. recent alteration in nutritional state, corticosteroid therapy, etc.). Reference values can also be important in a follow-up perspective, for the understanding of subsequent alteration in respiratory status, or the evaluation of therapeutic intervention. From the pathophysiological point of view, it is trivial to say that exploring respiratory muscles is useful for a better understanding of disease and symptoms. This is strongly linked to the clinical aspect, because potential therapeutic intervention should rely on detailed physiological data.

Increased airway resistance to airflow is the hallmark of COPD. Although the primary phenomenon is expiratory flow limitation, the disease and some compensatory mechanisms are associated with a predominantly inspiratory load. For example, inspiratory time is shortened to allow more time for exhalation, which increases the energy demand to inspiratory muscles. Because of air-trapping, and possibly to take advantage of an increased recoil pressure decreasing airway collapsibility, COPD patients breathe at high lung volumes. Such hyperinflation results in passive shortening of inspiratory muscles, and, in the absence of compensatory mechanisms, would lead to force reduction and decreased ability to generate negative pleural pressure. Besides length reduction, hyperinflation affects diaphragm shape and its geometrical relationship with the rib cage, modifying the transformation of force into pressure. What the changes in diaphragm shape are during chronic hyperinflation and how important they are, is not yet known.
Because COPD not only makes it harder to breathe but also impairs the capacity of the diaphragm to handle the load, diaphragm dysfunction is a good candidate among determinants of patients' respiratory handicap. However, demonstrating the benefit of therapeutic interventions aimed at diaphragm strength improvement or diaphragm rest has proved difficult [2]. This can be partly due, specific tests being difficult to use, to trials often looking at end-points to which respiratory muscle are only partial contributors.

When diaphragm specific investigation techniques have been applied to severely obstructive and hyperinflated well-nourished COPD patients, they have shown surprising results [3]. Transdiaphragmatic pressure (Pdi) response to bilateral phrenic nerve stimulation at functional residual capacity (FRC) was indeed reduced as compared with controls (16.9 vs $26.2 \mathrm{cmH}_{2} \mathrm{O}$ on average). However, the proportion of Pdi which was transformed into a useful fall in pleural pressure $(\mathrm{Ppl})$ was overpreserved (50 vs 43\%). In controls, acute increase in lung volume resulted in a dramatic fall both in Pdi and in the oesophageal pressure (Poes) to Pdi ratio. At all lung volumes above their FRC, COPD patients outperformed healthy subjects with respect to these two parameters. The patients studied were able to voluntarily produce a maximal diaphragmatic contraction, as demonstrated by the twitch occlusion technique. The relative preservation of diaphragmatic pressure generating ability, in circumstances where a marked reduction was predictable, indicates efficient compensatory mechanisms.

In animal models of elastase-induced emphysema, a phenomenon of length adaptation has been demonstrated to explain similar results: muscle fibres which are put at lengths shorter than optimal for long periods, lose sarcomeres [4]. As a consequence, the force-length relationship of the muscle is shifted, so that identical tension is generated at shorter length. Another hypothesis that can be called upon in COPD patients is decreased rib cage distortability, which would explain maintained pressure generation in spite of reduced force. It should be pointed out that compensation of diaphragmatic disadvantage, if present, is imperfect. In fact, the pressure generating advantage of the diaphragm in emphysema is manifest only at volumes above those at which natural spontaneous breathing occurs. Also, preserved capacity to generate pressure does not necessarily correspond to ability to displace an adequate tidal volume, which would be the ultimate target of ideal compensation [5].

\section{Short appraisal of "classical" and current investigatory techniques}

Any technique aimed at determining the state of an active system includes two aspects. The first is the nature of the output studied (e.g. for respiratory muscles, electromyography, pressure generation, or volume displacement). The second is the state of activity of the system at the time it is assessed, and the nature of the stimulus to test it. 


\section{Respiratory muscle output}

Volume displacement. Volume displacement can be considered as the final goal of inspiratory muscle action; and, therefore, could be the interesting parameter to look at. However, volume displacement is at the very end of the sequence of events described in figure 1. Therefore, volumes can only serve as a gross index of inspiratory muscle capacity. For instance, inspiratory capacity (IC) is often used as a screening test in difficult settings, such as the Intensive Care Unit, e.g. in order to assess weaning possibilities, or to follow-up the course of neuromuscular diseases. Although normal IC values are probably associated with minor muscle dysfunction, low values are nondiscriminative of the source of the problem. In the setting of the stable COPD patients, IC appears of limited interest with respect to muscle function per se.

Pressure generation. For volume to be driven, inspiratory muscle force has to be transformed into negative Ppl. Negative mouth pressure $(\mathrm{Pm})$ reflects the aggregate action of various inspiratory muscles. It has been shown that it could be brought down to levels close to normal in spite of the absence or quasi-absence of diaphragm contraction. Therefore, if, and only if, it adequately reflects $\mathrm{Ppl}$ (see below), Pm provides no more than a global inspiratory index. Pdi, obtained as the difference in pressure above and below the contracting diaphragm, has the advantage of being specific of it. Indeed, the diaphragm is the only muscle that can raise abdominal pressure whilst lowering pleural pressure. It must be emphasized that if Pdi is the net result of diaphragm contraction, only the part of it that is transformed in negative $\mathrm{Ppl}$ is useful to inspiration. The Poes to Pdi ratio can, thus, be viewed as a reflection of diaphragmatic inspiratory action. Unfortunately, measuring Pdi requires the use of two balloon catheters, one positioned in the oesophagus, and the other in the stomach. This is not an easy and short procedure, and methodological mistakes are possible. It is often ill-tolerated by patients.

Pressure depends on tension, which depends on length, which is strongly affected by lung volume. It must be emphasized that, because "stable" does not mean "immutable", pressure measurement for follow-up purposes in COPD patients must be associated with a reproducible measurement of lung volume. Short-term changes in the degree of hyperinflation, even of small magnitude, can markedly affect the ability of the diaphragm to produce a negative Ppl. In our view, correct interpretation of precise indices of diaphragmatic function should systematically be associated with a simultaneous FRC measurement. For less specific and rapid indices, a simple way to take volume into account is to use a pressure-volume envelope (Rahn diagram) built with the easily reproducible points (i.e. total lung capacity and residual volume).

Electromyography (EMG). EMG is interesting, in that it provides information on muscle function that directly relates to its intrinsic contractile properties. It is the reference means of evaluating muscle activity, and integrated EMG is proportional to muscle force. However, several technical drawbacks limit its clinical use for diaphragm assessment. During spontaneous respiration, surface electrodes placed on the chest at the 6-7th right and 7-8th left intercostal spaces record the summation of electrical activities arising from the diaphragm, but also from intercostal muscles and abdominal muscles. Also, lung volume, electrode position, and other factors have been shown to affect the EMG signal independently of muscle state. Day-to-day reproducibility is low and unpredictable.

\section{State of activity of the system}

Muscles are effectors of neural commands. They deliver outputs that directly depend on the intensity of the command, which is generally unknown or variable. Data provided by respiratory muscle testing during voluntary efforts are highly dependent on subject cooperation and can be improved by feedback. Furthermore, even in co-operating subjects, the respective contribution of muscle groups to a given output is unpredictable. As for inspiratory capacity (see above), normal values obtained during static voluntary manoeuvres probably point to the absence of clinically relevant muscle dysfunction, but abnormal values can have multiple sources. Therefore, static manoeuvres seem useful at a given moment, the clinician being able to evaluate his patient's status with respect to infection and nutrition, as well as his degree of co-operation. They are probably difficult to use for follow-up purposes. Simplification of devices used to measure them should promote their use as screening tests [6].

Dynamic maximal voluntary contractions, such as the sniff-test, represent attempts to standardize the neural stimulus to the muscles [7]. Different outputs can be studied, including noninvasive ones [8]. Although reported results on reproducibility are controversial, dynamic maximal voluntary contractions certainly represent a useful tool in patients capable of being trained, and if some feedback is used. Contrary to what is the case for static manoeuvres, no large series of data obtained in stable COPD patients is available to define normal values.

Supramaximal phrenic nerve stimulation (PNS) produces uniform contractions of the entire diaphragm (twitches) independent of any spontaneous or voluntary effort. Used in isolation, PNS provides information about the conjunction of contractile properties of the diaphragm [9], mechanical properties of the chest wall, and diaphragm-rib cage interactions. Superimposed upon naturally occurring or voluntary contractions, it helps to distinguish the intrinsic function of the diaphragm from the level of the diaphragm activation by the central nervous system (twitch occlusion principle) [9]. Because of the unicity of the phrenic-diaphragm relationship, (the diaphragm is the only muscle that contracts after phrenic stimulation) PNS ascertains the diaphragmatic origin of surface signals such as EMG, and the exclusive diaphragm origin of otherwise nonspecific signals, e.g. Poes or Pm (see below). 
In terms of reproducibility, suppressing voluntary influence and knowing the characteristics of the stimulus are major advantages of the technique. They are counterbalanced by some inconveniences. The twitch contraction is far from realistic: spontaneous contractions arise from trains of commands of graded intensity resulting in progressive fibre recruitment, rather than from simultaneous supramaximal activation. When stimulation is selective for the phrenic nerve (transcutaneous electrical stimulation), the resulting diaphragm contraction interacts with a relaxed and highly distortable rib cage. This is not the case during normal respiration. It has been shown in normal subjects that the small distortions accompanying a spontaneous breath contribute significantly to diaphragmatic inflationary force that is transmitted via the upper rib cage to the lung. In COPD patients, where neck muscles are often hypertrophic and where the pattern of inspiratory muscle recruitment is very different from that in normal subjects, this could be an important confounding factor. From a practical point of view, electrical stimulation (ES) of the phrenic nerve has not only to be supramaximal, but also bilateral. Unilateral stimulation induces major rib cage distortion that makes the resulting pressure output impossible to interpret [10]. Therefore, ES is not an easy technique to perform, and the operator must be highly trained for the results to be relied upon. Current intensities required to achieve supramaximal activation can exceed $40-50 \mathrm{~mA}$, and can at times be perceived as too painful by some patients. As will be discussed below, cervical magnetic stimulation (CMS) [11] solves some of these problems by being painless, easier to perform, and perhaps "closer to life", because it stabilizes the rib cage by co-activating upper rib cage and other muscles. Its lack of specifity has at times cast doubt about its value as a pure diaphragm tool.

\section{Perspectives}

\section{Criteria for an ideal technique}

Such a technique should be easy to perform. A pulmonary function test (PFT) laboratory technician should be able to operate the technique after a reasonably short training period. The test should be well-tolerated and short enough in duration, in order to allow large studies of out-patients who are not particularly motivated as well as repeated measures over time in a given patient.

The results of the test should be as independent as possible of patient co-operation. At best, they should relate to intrinsic muscle properties alone, that is to say be devoid of influence of lung volume, or rib cage properties, or diaphragm-rib cage interaction.

Phrenic nerve stimulation: can it be easier to perform and less invasive?

Among available tests, PNS is probably one of the closest to the ideal criteria. As pointed out earlier, PNS in its current state has limitations that have probably prevented it from becoming a routine technique and have confined it to research laboratories and limited series. It has been shown that magnetic stimulation applied over the vertebral column at the level of the spinous process of the $5-7$ th cervical vertebra resulted in bilateral supramaximal diaphragm response, as illustrated by surface EMG and Pdi signals [11]. Based on the principle of nerve stimulation by secondary currents induced by a powerful magnetic field, magnetic stimulation is painless and devoid of adverse effects. CMS is easy to perform. Using a single $9-10 \mathrm{~cm}$ coil, an operator can obtain and reproduce bilateral PNS using one hand. The training time to achieve a satisfactory and reproducible stimulation is very short, generally less than 15 min. Recent reports suggest that CMS reproducibility in a given individual is slightly better than that of classical electrical bilateral stimulation [12].

However, the use of CMS to investigate diaphragmatic function has, up to now, been somewhat controversial, essentially because the diaphragm is not the only muscle activated by the stimulus. Indeed, after magnetic stimulation Pdi twitch is greater than after electrical stimulation, the difference being accounted for by a greater oesophageal pressure swing. A direct contribution of extra-diaphragmatic muscles to the Ppl swing would limit the usefulness of the technique as a diaphragm tool. However, it is highly likely that the aforementioned difference is due rather to rib cage muscle being active at the time when CMS induced diaphragmatic contraction occurs, making the rib cage less distortable [13]. In any event, these considerations are probably of little relevance from a clinical, follow-up, point of view.

Currently, CMS is only beginning to be used in the clinical setting for respiratory applications (its use has recently contributed to the elucidation of some of the mechanisms responsible for difficult weaning mechanical ventilation after cardiac surgery [14]), and there are as yet no data in COPD patients. Clearly, progress in the understanding of the respective meanings of classical PNS and CMS data are needed, and so are applications to patients.

Assessment of diaphragm output: can it become easier and less invasive?

Mouth pressure. The two-balloons technique to measure Pdi is an invasive one, but Pm measured during voluntary manoeuvres is not a reliable index of diaphragm function. Associating Pm to phrenic stimulation would have the advantage of being noninvasive and not dependent on subject co-operation, and would give information about the inspiratory action of a pure diaphragmatic contraction. This is subject to Pm adequately reflecting $\mathrm{Ppl}$, a condition requiring that the time constant for equilibration between mouth and alveolar pressure be short relative to the time during which Poes changes. During isovolumetric twitches in normal subjects or patients with normal lungs but suspected 
diaphragm weakness, it has been shown that it is indeed the case, whether the stimulation is electrical [15] or magnetic (S. Hamnergard, J. Moxham and M. Green, personal communication). Pm twitch correlates well with Poes twitch, at relaxed FRC and relaxed volumes above FRC. It has been shown that it could provide a satisfactory index of acute diaphragmatic fatigue in normals [15].

In COPD patients, pressure equilibration between the alveoli and mouth is incomplete due to the airways acting as a resistance-capacitance (R-C) filter between the two. Because airways time constant is long and diaphragmatic contraction time short, twitch Pm appears damped and time-lagged with respect to Poes. However, when the subjects are instructed to generate a gentle inspiratory effort upon which stimulation is superimposed, Pm and Poes match perfectly [16]. The reason for this is not clear, but can be related to decreased upper airway compliance or more uniform pleural pressure during the Müller manoeuvre, because of smaller rib cage deformation. If this is true, in such patients CMS would be a better technique to assess mouth pressure response to diaphragmatic contraction than electrical stimulation.

In severe COPD patients with twitch Pdi at relaxed FRC of about $16 \mathrm{cmH}_{2} \mathrm{O}$ and a Poes:Pdi ratio of about $50 \%, \mathrm{Pm}$ twitch has been estimated at $4 \mathrm{cmH}_{2} \mathrm{O}$ in relaxed condition (about one half of Poes), but became equal to Poes when stimulations were superimposed upon diaphragmatic contractions. Application to Pm of the twitch occlusion principle has allowed prediction of twitch Pm at relaxed FRC and of maximal diaphragm related Pm.

Nostril pressure. Results similar to those with Pm have been reported during the sniff test with pressure measured at the nostril [17]. In this technique, one nostril is plugged, the pressure is measured through the plug whilst the subject performs a maximal sniff via the free nostril. Such a combination of a very simple pressure measurement to a relatively easy to perform and reproducible contraction, if validated in the setting of airway obstruction, will probably provide a powerful follow-up tool.

Phonomyogram. When skeletal muscles contract, they produce vibrations and low frequency sounds easily recorded via a microphone taped on the skin. This mechanical signal is called phonomyogram (PMG). PMG provides an index of isometric individual muscle force and of motor unit recruitment. During electrostimulation, there is a significant correlation between PMG and current pulse intensity. During voluntary movements, PMG is correlated with biomechanical quantities, such as acceleration and external work. It can be used to evaluate the excitation-contraction coupling during fatigue: during maximal voluntary static contractions maintained up to a $50 \%$ reduction of their initial force, integrated PMG decreases in parallel with force [18], whereas integrated EMG is often not adequate to monitor fatigue.

Diaphragm contraction is associated with a PMG signal. As for surface EMG signals, the surface PMG signals recorded during spontaneous breathing represent a summation of various muscle activities. The problem can be bypassed by use of phrenic nerve stimulation. Because PNS related PMG would directly reflect diaphragmatic force, the need for bilateral stimulation would be alleviated, and a combination of PMG with unilateral electrical stimulation would suffice to assess diaphragmatic contractility. Preliminary data seem promising [19].

\section{Conclusion}

Although the pathophysiology of respiratory muscle involvement in COPD is better understood, precise clinical investigation of respiratory muscle function in such patients remains at its infant stage. It is likely that technological advances oriented toward less invasivity and greater ease of use will help change this situation. For example, combination of mouth pressure measurement with CMS, or unilateral electrical phrenic stimulation with homolateral PMG are highly promising.

It should be kept in mind that the clinical investigation of respiratory muscle function, and particularly of the diaphragm, cannot rely upon one single, universal test that would fulfil criteria of acceptability and quality. Rather, clinicians will have to use a limited panel of tests, with their respective strengths, that will both allow easy and efficient follow-up of diaphragm function in COPD, and improve the rationale and quality of clinical interventions in this setting.

\section{References}

1. Rochester DF. The respiratory muscles in COPD: state of the art. Chest 1984; 85: 47S-50S.

2. Shapiro SH, Ernst P, Gray-Donald K, et al. Effect of negative pressure ventilation in severe chronic obstructive pulmonary disease. Lancet 1992; 340: 14251429.

3. Similowski T, Yan S, Gauthier AP, Macklem PT, Bellemare F. Contractile properties of the human diaphragm during chronic hyperinflation. N Engl J Med 1991; 325: 917-923.

4. Farkas GA, Roussos C. Diaphragm in emphysematous hamsters: sarcoma adaptability. J Appl Physiol: Respirat Environ Exercise Physiol 1983; 54: 1635-1640.

5. Rochester DF. The diaphragm in COPD: better than expected, but not good enough. N Engl J Med 1991; 325: 961-962.

6. Hamnegard CH, Wragg S, Kyroussis D, Aquilina R, Moxham J, Green M. Portable measurement of maximum mouth pressures. Eur Respir J 1994; 7: 398-401.

7. Miller JM, Moxham J, Green M, The maximal sniff in the assessment of diaphragm function in man, Clin Sci 1985; 69: 91-96.

8. Koulouris N, Mulvey DA, Laroche CA, Sawicka EH, Green M, Moxham J, The measurement of inspiratory muscle strength by sniff esophageal, nasopharyngeal, and mouth pressures. Am Rev Respir Dis 1989; 139: 641-646.

9. Bellemare F, Bigland-Ritchie B. Assessment of human diapragmatic strength and activation using phrenic nerve stimulation. Respir Physiol 1984; 58: 263-277. 
10. Bellemare F, Bigland-Ritchie B, Woods JJ. Contractile properties of the human diaphragm in vivo. J Appl Physiol 1986; 61: 1153-1161.

11. Similowski T, Fleury B, Launois S, Cathala HP, Bouche P, Derenne JP. Cervical magnetic stimulation: a new and painless method for bilateral phrenic nerve stimulation in conscious humans. J Appl Physiol 1989; 67: 1311-1318.

12. Wragg S, Aquilina $\mathrm{R}$, Moran $\mathrm{J}$, et al. A comparison of cervical magnetic stimulation and bilateral percutaneous electrical stimulation of the phrenic nerves in normal subjects. Eur Respir J 1994; 7: 1788-1992.

13. Similowski T, Launois S, Whitelaw WA, Derenne J-P. Origin and characteristics of diaphragm contraction induced by cervical magnetic stimulation. Am Rev Respir Dis 1993; 147: A699 (Abstract).

14. Diehl JL, Lofaso F, Deleuze P, Similowski T, Lemaire F, Brochard L. Clinically relevant diaphragmatic dysfunction after cardiac operations. J Thorac Cardiovasc Surg 1994; 107: 487-498.
15. Yans S, Gauthier AP, Similowski T, Macklem PT, Bellemare F. Evaluation of human diaphragm contractility using mouth pressure twitches. Am Rev Respir Dis 1992; 145: 1064-1069.

16. Similowski T, Yan S, Gauthier AP, Bellemare F, Macklem PT. Assessment of diaphragm function using mouth pressure twitches in COPD patients. Am Rev Respir Dis 1993; 147: 850-856.

17. Heritier F, Rahm F, Pasche P, Fitting JW. Sniff nasal inspiratory pressure: a noninvasive assessment of inspiratory muscle strength. AmJ Respir Crit Care Med (in press).

18. Maton B, Petitjean M, Cnockaert JC. Phonomyogram and electromyogram relationships with isometric force reinvestigated in man. Eur J Appl Physiol 1990; 60: 194-201.

19. Similowski T. Petitjean M, Maton B, Monod H, Derenne J-P. Phonomyogram of the diaphragm during phrenic stimulation and sniff-test. Am Rev Respir Dis 1992; 145: A255 (Abstract). 